\title{
New quantum phases in a one-dimensional Josephson array
}

\author{
A.I. Larkin ${ }^{1,2}$, L.I. Glazman ${ }^{1}$ \\ ${ }^{1}$ Theoretical Physics Institute, University of Minnesota, Minneapolis, MN 55455 \\ ${ }^{2}$ L.D. Landau Institute for Theoretical Physics, 117940 Moscow, Russia
}

(Draft of October 11, 2018)

\begin{abstract}
We examine the phase diagram of an ordered one-dimensional Josephson array of small grains. The average grain charge in such a system can be tuned by means of gate voltage. At small grain-tograin conductance, this system is strongly correlated because of the charge discreteness constraint (Coulomb blockade). At the gate voltages in the vicinity of the charge degeneracy points, we find new phases equivalent to a commensurate charge density wave and to a repulsive Luttinger liquid. The existence of these phases can be probed through a special dependence of the Josephson current on the gate voltage.
\end{abstract}

PACS numbers: 73.20.Fz,03.65.Sq, 05.45.+b

In the physics of phase transitions, the special class of transitions occuring at zero temperature have attracted significant attention in the recent years [1]. At zero temperature, long-range order may be destroyed by quantum fluctuations. In some systems, the magnitude of these fluctuations is controlled by an external parameter. Therefore such a system may undergo transitions between the phases with qualitatively different behavior of long-range correlations. In contrast to the conventional finite-temperature transitions, the quantum transition at $T=0$ can occur even in a one-dimensional system. For example, as it was predicted by Bradley and Doniach [2], a one-dimensional array of superconducting grains linked by Josephson junctions may behave as a superconductor or insulator depending on the ratio of charging and Josephson energies. Recently a special sample design allowing one to vary continuously the strength of inter-grain Josephson junctions was developed [3] and an attempt to examine the vicinity of the quantum phase transition was undertaken [4].

Paper [2] and the majority of later theoretical papers devoted to the one-dimensional Josephson junction arrays, assumed that the grains of an array are tuned to the point of particle-hole symmetry. In this case, the transition from a superconductor to Mott insulator is of the Berezinskii-Kosterlitz-Thouless (BKT) type [2]. Within a broader framework of quantum phase transitions in a system of interacting bosons [5], it became clear that breaking the particle-hole symmetry immediately changes the universality class of the transition.

Much of the physics of interacting bosons can be applied directly to the Josephson junction arrays. For an array, a boson is replaced by a Cooper pair. Breaking the particle-hole symmetry means simply applying some voltage $V_{g}$ to a gate which is electrostatically coupled to all grains of the array. Breaking the symmetry leads to a symmetric in $V_{g}$ shift of the superconductor-insulator transition. Recently [6] some experimental evidence was found for such a symmetric shift for two-dimensional sys- tems.

In this paper, we found new, compared to the predicted in [5], phases of a one-dimensional Josephson array. These appear due to the effective repulsion between Cooper pairs at different sites of the array. In the plane of controlling parameters, which are the Josephson energy $E_{J}$ and voltage $V_{g}$, these phases occupy the vicinities of the charge degeneracy points. At $E_{J}=0$ the effective repulsion leads to a period-doubling transition. It separates the known Mott phase with homogeneously distributed Cooper pairs, from a new phase with alternate occupation of the grains ("Neel phase"). As $E_{J}$ increases, the regions of Mott and Neel phases separate from each other, giving way to another, intermediate phase. This phase is equivalent to a Luttinger liquid with repulsion. Further increase of the Josephson coupling brings the system to a transition into the superconducting state (Luttinger liquid with attraction). We found the corresponding transition line on the phase diagram $\left\{E_{J}, V_{g}\right\}$, see Fig. 1. This line can be identified with an abrupt change in the behavior of the Josephson current through a chain containing one weaker link.

We also found the critical exponents for the transition line between the Luttinger liquid and Mott phase.

To start with, we consider a one-dimensional Josephson array in the absence of Cooper pair repulsion. The simplest Hamiltonian that allows for the superconductorinsulator phase transition, has the form:

$$
\begin{aligned}
H=H_{C}+H_{J}, \quad H_{C} & =\frac{E_{C}}{2} \sum_{i}\left(\frac{\partial}{i \partial \phi_{i}}-\frac{\mathcal{N}}{2}\right)^{2}, \\
H_{J} & =-E_{J} \sum_{i} \cos \left(\phi_{i+1}-\phi_{i}\right) .
\end{aligned}
$$

In comparison with [2, 7, 8], Hamiltonian (11) accounts for the influence of the gate voltage, $e \mathcal{N} \propto V_{g}$ (in the absence of charge quantization, $e \mathcal{N}$ is the average grain charge induced by the gate); $E_{C}$ is the charging energy of a grain. 
The two parts of the Hamiltonian (11) do not commute with each other, leading to quantum fluctuations of phase $\phi_{i}$ of the superconducting order parameter in each grain. To start with, we consider the domain of weak Josephson coupling, $E_{J} \ll E_{C}$, where the fluctuations are strong. In the limit $E_{J} \rightarrow 0$, the Josephson array is a Mott insulator at almost any $\mathcal{N}$. The exceptions are the discrete points $\mathcal{N}=2 n+1$, where a grain with charges $2 n e$ and $2(n+1) e$ has the same energies. Josephson tunneling lifts this degeneracy. To analyze the properties of the array in the vicinity of the degeneracy points, we can project the Hamiltonian (1) on the subspace of states with charges of each grain confined to the values $2 n e$ and $2(n+1) e$ only. In the lowest order in $E_{J}$ the projected Hamiltonian has the form:

$$
\begin{array}{r}
H_{C}^{(0)}=\frac{E_{C}}{2} \sum_{i}\left(\sigma_{i}^{z}-h\right)^{2}, \\
H_{J}^{(0)}=\frac{E_{J}}{2} \sum_{i}\left(\sigma_{i}^{+} \sigma_{i+1}^{-}+\sigma_{i}^{-} \sigma_{i+1}^{+}\right),
\end{array}
$$

where $\sigma^{z}, \sigma^{+}, \sigma^{-}$are the Pauli matrices, and the "magnetic field" $h=(\mathcal{N}-2 n-1) / 2$ allows for tuning the system to a degeneracy point by means of the gate voltage. The Hamiltonian (2) can be converted to the conventional tight-binding Hamiltonian for free fermions by means of the Jordan-Wigner transformation, and then diagonalized [9] in the plane wave representation:

$$
H_{0}=\sum_{k} \varepsilon_{k} a_{k}^{\dagger} a_{k}, \quad \varepsilon_{k}=E_{J} \cos k-E_{C} h .
$$

The fermion band is completely full or totally empty, if $|h|>E_{J} / E_{C}$. This condition defines the location of the Mott phase in the plane of dimensionless parameters $\left\{\mathcal{N}, E_{J} / E_{C}\right\}$. The Mott gap, $\epsilon_{M}=\left|E_{C}\right| h\left|-E_{J}\right|$, coincides with the lowest possible excitation energy, and vanishes at the phase boundary. Clearly, the phase diagram is periodic in $\mathcal{N}$ with period 2 . This allows us to consider further only a "strip" of the phase diagram, $0 \leq \mathcal{N} \leq 2$ which corresponds to $n=0$ in the above formulas.

The lowest order in $E_{J}$ we used is sufficient to find the characteristics of the Mott phase. The complementary phase in this approximation is equivalent to a noninteracting Fermi gas. It is known however, that even a weak interaction is relevant in one dimension, transforming the gas into a Luttinger liquid. There are two competing mechanisms of interaction between the fermions.

The inter-grain electrostatic interaction leads to an effective repulsion between the Cooper pairs. We will concentrate on the case of a relatively weak inter-grain interaction, $E_{z} \ll E_{C}$, which allows us to use Hamiltonian (2) as a starting point. In a realistic system with a gate, the interaction between distant grains is screened out, and only the nearest-neighbor term should be retained:

$$
H_{z}=\sum_{i} E_{z} \sigma_{i}^{z} \sigma_{i+1}^{z}
$$

On the other hand, the higher order expansion in $E_{J} / E_{C}$ leads to an effective attraction between the JordanWigner fermions. To find the corresponding correction to the low-energy Hamiltonian (2), we should take into account the virtual states with energies exceeding $E_{C}$. To do this, we use the perturbation theory. In the secondorder perturbation theory, the effective Hamiltonian reduced to the subspace of charges 0,2 , takes the form:

$$
H_{2}=P H_{J}(1-P) \frac{1}{\varepsilon-H_{C}} H_{J} P
$$

Here $P=\prod_{i} P_{i}$, and $P_{i}$ is the projection operator on the subspace of states with charges 0 and 2 for each grain; energy $\varepsilon$ belongs to the reduced energy band, and may be neglected in comparison with $E_{C}$. To implement the projection procedure in (5), we use the identities, $P_{i} \exp \left( \pm \phi_{i}\right)\left(1-P_{i}\right) \exp \left(\mp \phi_{i}\right) P_{i}=\left(1 \mp \sigma_{i}^{z}\right) / 2$, that can be easily checked. Thus, the result of projection can be written in terms of Pauli matrices,

$H_{2}=-\frac{E_{J}^{2}}{4 E_{C}} \sum_{i}\left(\frac{3}{4} \sigma_{i}^{z} \sigma_{i+1}^{z}+\sigma_{i+1}^{+} \sigma_{i-1}^{-}+\sigma_{i-1}^{+} \sigma_{i+1}^{-}\right)$.

The full Hamiltonian $H^{(0)}+H_{z}+H_{2}$ enables us to investigate the phase diagram in the vicinity of the charge degeneracy line $(h \ll 1)$.

We can neglect the interaction $H_{2}$, if $E_{J}^{2} / E_{C} E_{z} \ll 1$; in this limit, our system with Hamiltonian $H^{(0)}+H_{z}$ is equivalent to a one-dimensional anisotropic Heisenberg model in a magnetic field. It is known 10 that at $E_{J}<4 E_{z}$ and relatively small field $|h|<h_{1}$, the antiferromagnetic Izing interaction dominates the physics of the anisotropic Hesenberg model, whereas in a large field, $|h|>h_{2}$, the chain is in the ferromagnetic state. Returning to the language of the initial chain of grains, the ferromagnetic phase corresponds to Mott insulator. The low-field Neel phase is a commensurate charge density wave state with period 2 (on average, every second grain of the chain is occupied by a Cooper pair). In both states, there is a gap for excitations. The region in between, $h_{1}<|h|<h_{2}$, is occupied by Luttinger liquid. The width of the Luttinger liquid region depends on the value of $E_{J}$. In the limiting case $E_{J}=0$ which corresponds to the Izing model, this width shrinks to zero. At small $E_{J}$, the dependence of both $h_{1}$ and $h_{2}$ is linear in $E_{J}$ with coefficients of opposite sign [10], and the width of the Luttinger liquid region is proportional to $E_{J}$. The field $h_{1}$ turns zero for an isotropic antiferromagnet, $E_{J}=4 E_{C}$. At larger $E_{J}$, the Neel state is absent, and the liquid phase exists even at $h=0$, see Fig. 1 .

With the further increase of $E_{J}$, the interaction $H_{2}$ becomes important. As we will see, it alters the sign of 
interaction in the Luttinger liquid. To study the interaction, we transform the spin Hamiltonian $H_{z}+H_{2}$ to the Jordan-Wigner fermion representation. In addition to quadratic in $a, a^{\dagger}$ terms, this Hamiltonian gives rise also to two-body interaction $V_{\text {int }}$. The significant part of (weak) interaction $V_{\text {int }}$ that defines the properties of Luttinger liquid, corresponds to the interaction between the left- and right-movers. Near the one-dimensional Fermi "surface" $k= \pm k_{F}$ this part can be written as:

$$
\begin{aligned}
& V_{\mathrm{int}}=E_{J} \lambda \sum_{k_{1}, k_{2}, q} \sin ^{2} k_{F} a_{k_{1}}^{\dagger} a_{k_{2}}^{\dagger} a_{k_{2}+q} a_{k_{1}-q}, \\
& \lambda=\left(\frac{8 E_{z}}{E_{J}}-\frac{7 E_{J}}{2 E_{C}}\right) .
\end{aligned}
$$

Here $k_{1} \approx k_{F}, k_{2} \approx-k_{F}$, and $q \ll k_{F}$; the Fermi wavevector varies with the gate voltage.

Interaction $(7)$ vanishes at the phase boundary of the Luttinger liquid with Mott phase $\left(k_{F} \rightarrow 0\right.$ or $\left.k_{F} \rightarrow \pi\right)$, regardless the relations between parameters $E_{z}, E_{J}$, and $E_{C}$. Therefore, we can use the free-fermion model to study the critical behavior of our system near the phase boundary. The divergent length scale, $\xi(h) \sim 1 / k_{F}$, characterizes the distance between free charges in the conducting phase. Using Eq. (3), we find $\xi \propto \delta^{-1 / 2}$, so that the critical exponent $\nu$ equals $1 / 2$ (here $\delta=h_{2}-|h|$ is the distance to the phase boundary). The characterisitic frequency scale $\Omega$ is given simply by the Fermi energy. The corresponding exponent $z$, defined by the relation $\Omega \sim \delta^{z \nu}$, is $z=2$. Finally the superfluid density [5], $\rho_{s} \propto \delta^{\zeta}$, should be identified with the density of fermions $\rho_{s} \sim \delta^{1 / 2}$, which determines $\zeta=1 / 2$.

Vanishing of the interaction at the phase boundary with Mott phase is a property far more general than the result of perturbation theory (7), and it reflects the fact that we deal with spinless fermions. In the higher orders in $E_{J} / E_{C}$ the dependence of $h_{2}$ on $E_{J}$ becomes nonlinear, and the spectrum of fermions changes, but the critical exponents we found are not affected as long as $\mathcal{N} \neq 0,2$. The lines $\mathcal{N}=0,2$ are the lines of particle-hole symmetry. Because of this symmetry, the system belongs to a different universality class [5], and the transition to the Mott phase (which occurs at $E_{J} / E_{C} \approx 4 / \pi^{2}$ ) is of the BKT type.

Now we discuss the properties of the Josephson junctions chain in the Luttinger liquid state, which lowenergy properties are described by a quadratic Lagrangian [11, 12],

$$
\mathcal{L}=\frac{g}{2 \pi}\left[v\left(\partial_{x} \phi\right)^{2}+v^{-1}\left(\partial_{\tau} \phi\right)^{2}\right] .
$$

Here $v$ is the velocity of the acoustic excitations in the system (plasmons in our case), and $g$ determines the long-range behavior of all correlation functions. The phenomenological constant $g$ can be calculated in terms of the microscopic parameters in some limiting cases.

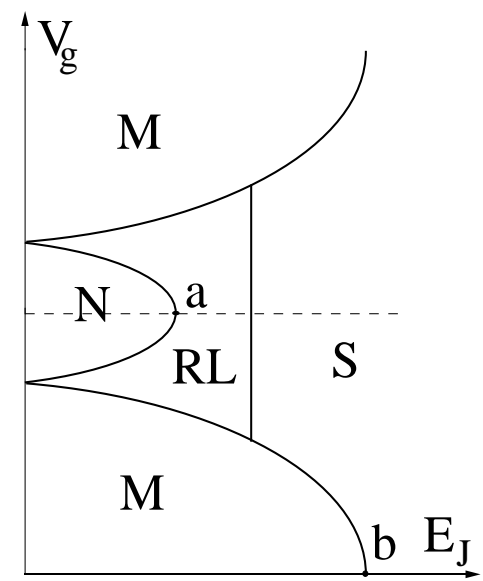

FIG. 1. Zero-temperature phases of the one-dimensional array of Josephson junctions controlled by the gate voltage $\left(V_{g}\right)$ and Josephson energy $\left(E_{J}\right)$. In addition to the known $[5$ Mott phase $M$ and superconducting phase $S$ (which is equivalent to the attractive Luttinger liquid), we find the commensurate charge density wave state $N$, and repulsive Luttinger liquid phase $R L$. The Luttinger liquid parameter $g$ equals 1 at the $R L-S$ boundary, and also at the boundary of the Mott phase; $g=1 / 4$ at $R L-N$ boundary. The special points on the phase transition line where the charge degeneracy occurs $(a)$, and where the particle-hole symmetry is observed (b), are multicritical points with $g=1 / 2$ and $g=2$ respectively.

Deep in the superconducting region, $E_{J} \gg E_{C}$, one can compare Eq. (8) with the quadratic Lagrangian derived by expansion of (11) in $\phi$, to find $g \approx \pi\left(E_{J} / E_{C}\right)^{1 / 2}$. In the opposite limit of a relatively weak Josephson coupling, $E_{z} \ll E_{J} \ll E_{C}$, constant $g$ can be found with the help of (7), $g \approx 1-(\lambda / 2 \pi) \sin k_{F}$. In addition to these limiting expressions for $g$, we know its value at the phase boundaries. We already mentioned that at the boundary with the Mott phase the interaction is vanishing, which means $g=1$. The exceptions are the special multicritical points [5], where the boundary intersects with a particlehole symmetry line (point $b$ in Fig. 1). If point $b$ is crossed along the symmetry line, the transition is of the BKT type with $g=2$. In the vicinity of these points, $g$ rapidly varies from $g=1$ to $g=2$. With the help of Haldane's theory [13] for anizotropic $s=1 / 2$ Hezenberg model in a magnetic field, we find $g=1 / 4$ for the boundary between the Luttinger liquid and Neel phases. The exceptions are the multicritical points ( $a$ in Fig. 1) of charge degeneracy, $h=0$, where $g=1 / 2$. Similarly to the lines of particlehole symmetry, the phase transition at $h=0$ is of the BKT type, see, e.g., 14.

Different phases on the diagram Fig. 1 can be distinguished by the dependence of the critical Josephson current on the length of the system $L$. Clearly, the gap in the excitation spectrum existing in the insulating phases leads to an exponential suppression of the critical current with $L$; the corresponding correlation length diverges (with exponent $\nu$ ) at the transition line.

To characterize the Luttinger liquid state, we consider 
a chain of junctions connecting two massive superconducting leads, and containing just one especially weak link, $E_{w} \ll E_{J}$. Deep in the superconducting state $(g \gg 1)$ we can neglect with the quantum fluctuations. The energy of the system consisting of $L$ junctions of the nominal strength $E_{J}$ and one weak link equals:

$$
E(\phi)=\frac{E_{J}}{2} \frac{\left(\phi-\phi_{w}\right)^{2}}{L}-E_{w} \cos \phi_{w}
$$

Here $\phi$ is the phase difference applied to the leads, and $\phi_{w}$ is the phase difference accross the weak link; the energy (9) should be minimized with respect to $\phi_{w}$. Quantum fluctuations of phases $\phi_{i}$ at finite $g$ lead to zero-point motion of $\phi_{w}$ around some average value $\bar{\phi}_{w}$, which depends on the external phase $\phi$. Averaging over the fluctuations $\delta \phi_{w}=\phi_{w}-\bar{\phi}_{w}$ results in the energy functional of the form (9) with $E_{w}$ renormalized by a proper Debye-Waller factor, and $\phi_{w}$ replaced by $\bar{\phi}_{w}$, see, e.g., [15]. For a short array, the renormalized Josephson coupling constant is $E_{w}^{\mathrm{eff}} \simeq E_{w}(1 / L)^{1 / g}$. Energy (9) corresponds to two inductors in series. Therefore, the response of the system to the external phase is dominated by the weaker of two elements. If $g>1$ (weaker quantum fluctuations), the renormalized energy $E_{w}^{\mathrm{eff}}$ decreases with $L$ slower than the first term in Eq. (9). Consequently, at $L$ exceeding the crossover length, $L^{*} \simeq\left(E_{J} / E_{w}\right)^{g /(g-1)}$, the energy (9) is dominated by the first term with $\phi_{w}=2 \pi n$. The Josephson current $\propto \partial E / \partial \phi$ has a sawtooth dependence on phase, with the amplitude proportional to $E_{J} / L$. Thus, the effect of the weak link is "healed" over the distance $L^{*}$.

If $g<1$, the weak link energy $E_{w}^{\text {eff }}$ falls off faster than $1 / L$, and this link remains the weakest of the two inductors connected in series at any $L$; consequently, $\bar{\phi}_{w}=\phi$. Therefore, "healing" does not occur, and at any $L$ the chain behaves as a single Josephson junction of strength $E_{w}^{\text {eff }}$. The corresponding current-phase relation is $I \sim E_{w} L^{1 / g} \sin \phi$.

The line $g=1$ on the phase diagram may be viewed as a phase transition [16] between the superconducting and insulating phases in the following sense. In the limit $L \rightarrow \infty$, the inductance $\mathcal{L}$ of an array with a single weak link is insensitive to the weak link if $g>1$ (superconducting state), and $\mathcal{L} \propto L / E_{J}$. In the insulating state, the scaling of inductance with $L$ is $g$-dependent, $\mathcal{L} \propto L^{1 / g} / E_{w}$, with exponent $1 / g>1$ [17].

To facilitate the quantitative analysis of the phase diagram, we considered the limit $\Delta \gg E_{C} \gg E_{z}$. Eazing the first, and lifting the second of these two restrictions does not alter the diagram qialitatively. Moreover, in a real system with large inter-grain capacitance [4] the energies $E_{C}$ and $E_{z}$ are of the same order, and the domains of charge density wave and of the repulsive Luttinger liquid are not small. The phase diagram changes drastically only if the superconducting gap $\Delta$ becomes smaller than some critical value, at which the grains may carry single electrons in the ground state. The charge density wave state is destoyed then, and a phase appears with an odd charge on each grain. For this phase, the Josephson current scales exponentially with $L$, but there is no gap in the excitation spectrum due to the dense quasiparticle spectrum in each superconducting grain.

We assumed a perfect order in the system, which means there are no spatial variations of the parameters of the Hamiltonian (11). The validity of the above analysis requires that the Josephson junctions chain is shorter than the mean free path for a Cooper pair propagating along the chain.

In conclusion, we examined the zero-temperature phase diagram of a one-dimensional chain of Josephson junctions. We have shown that accounting for the repulsion between Cooper pairs occupying different grains leads to new phases, which are equivalent to the phases of one-dimensional spinless fermions with repulsion on a lattice. We have studied in detail the boundaries of the quantum phases.

We are grateful to I.L. Aleiner, F. Hekking, T. Giamarchi, S.M. Girvin, and D. Haviland for discussions and numerous references to papers on related subjects. This work is supported by NSF Grant No. DMR-9423244.

[1] S.L. Sondhi, et al Rev. Mod. Phys., 69, 315 (1997)

[2] R.M. Bradley, and S. Doniach Phys. Rev. B 30, 1138 (1984)

[3] D.B. Haviland, and P. Delsing, Phys. Rev. B 54, 6857 (1996)

[4] D.B. Haviland, and P. Delsing, Chalmers University Preprint, 1996

[5] M.P.A. Fisher, et al, Phys. Rev. B 40, 546 (1989)

[6] A.M. Goldman, private communication, 1997

[7] P.W. Anderson, in Lectures on Many Body Problem, edited by E.R. Cainiello (Academic, New York, 1964), Vol. 2, p. 127

[8] K.B. Efetov, Zh. Eksp. Teor. Fiz. 78, 17 (1980) [Sov. Phys. - JETP 51, 1015 (1980)]

[9] D.C. Mattis, The Theory of Magnetism (Springer-Verlag, Berlin, New York, 1988)

[10] M. Fowler, and M.W. Puga, Phys. Rev. B 18, 421 (1978)

[11] K.B. Efetov, and A.I. Larkin, Zh. Eksp. Teor. Fiz. 69, 764 (1975) [Sov. Phys. - JETP 42, 390 (1975)]

[12] F.D.M. Haldane, Journ. of Phys. C, 14, 2585 (1981)

[13] F.D.M. Haldane, Phys. Rev. Lett. 45, 1358 (1980)

[14] R. Shankar, Internat. Journ. Modern Phys. B 42371 (1990)

[15] F.W.J Hekking, and L.I. Glazman Phys. Rev. B 55, 6551 (1997)

[16] The special role of $g=1$ value was earlier pointed out for the problems of conductance of a Luttinger liquid, see C.L. Kane, and M.P.A. Fisher, Phys. Rev. B 46, 15233 (1992), and of tunneling with dissipation, see, e.g., reviews: A.J. Leggett et al, Rev. Mod. Phys. 59, 1 (1987); G. Schon, A. Zaikin, Phys. Reports, 98, 237 (1990), and references therein.

[17] A similar suppression of tunneling of a pinned incommen- 
